\title{
HgSe: Metal or Semiconductor?
}

\author{
K.-U. Gawlik, L. Kipp,* and M. Skibowski \\ Institut für Experimentalphysik, Universität Kiel, D-24098 Kiel, Germany \\ N. Orłowski and R. Manzke \\ Institut für Physik, Humboldt-Universität Berlin, D-10115 Berlin, Germany
}

(Received 13 August 1996)

\begin{abstract}
From magnetotransport measurements it is generally believed that $\mathrm{Hg}$-VI compounds show zero gap semiconducting behavior. Applying combined angle-resolved photoemission and inverse photoemission spectroscopy on $\mathrm{HgSe}(001) c(2 \times 2)$, we observe a positive fundamental gap of about $0.42 \mathrm{eV}$ and a surface related state close to the Fermi level above the conduction band minimum. Following the results of this direct determination of the $\vec{k}$-resolved band structure, previous experiments favoring zero gap models of Hg-VI compounds need to be reinterpreted. [S0031-9007(97)03026-3]

PACS numbers: 73.20.At, 79.60.Bm
\end{abstract}

Because of its technological interest for electro-optical devices, the $\mathrm{Zn}$ and Cd containing II-VI compound semiconductors have been studied intensively over the past decade. The group of $\mathrm{Hg}$ containing II-VI compounds, however, has been scarcely investigated since its electronic structures were reported to reveal zero or even negative fundamental gaps with inverted band structures. Following results on $\alpha$-Sn from Groves and Paul [1], the valence band maximum (VBM) was considered to be degenerate with the conduction band minimum $(\mathrm{CBM})$ revealing $\Gamma_{8}$ symmetry. Early magnetotransport measurements measuring extremal cross sections of Fermi surfaces indeed found evidence for an inverted band structure in bulk $\mathrm{HgSe}[2-$ 4] similar to those observed on $\mathrm{HgTe}$ [5] and $\beta$ - $\mathrm{HgS} \mathrm{[6].}$

Semiempirical band structure calculations for $\mathrm{HgSe}$ [7-9] and HgTe [7,8,10] fitting those data, consequently, show an inverted band structure with valence band widths of the order of 3.3-4.4 eV for $\mathrm{HgSe}$ and 3.6-4.8 eV for $\mathrm{HgTe}$, respectively. Photoemission results on $\mathrm{HgSe}$ and HgTe [11-15], in contrast, exhibit larger valence band widths of about 5.0-5.8 eV. The experimental position of the Fermi level with respect to the VBM is of particular interest in order to distinguish between metallic and semiconducting band structures. It has, however, only been reported for $\mathrm{HgTe}(110)$ by $\mathrm{Yu}$ et al. [11]. They determined the Fermi level to be $0.59 \mathrm{eV}$ above the VBM. Infrared absorption data [16,17] show two absorption edges around 0.4 and $0.2 \mathrm{eV}$ photon energy which are interpreted as transitions from the two upper valence bands near $\Gamma_{8}$ and $\Gamma_{6}$ into the $\Gamma_{8}$ conduction band, leading to a fundamental energy gap of approximately $-0.2 \mathrm{eV}$.

The experimental results, together with the semiempirical band structure calculations reported so far, do not give a consistent picture of the band structure around the Fermi level of Mercury containing II-VI compounds. This can be attributed to the type of experiments giving only indirect information on band structures (optical and magnetotransport measurements) and theories fitting these data.
In this Letter we take $\mathrm{HgSe}$ as the prototype material of $\mathrm{Hg}$-VI compounds which, in addition, may be compared to the better-known narrow gap semiconductor InAs having a similar lattice constant. We report on direct measurements of the $k$-resolved occupied and unoccupied band structure around the Fermi level of $\mathrm{HgSe}(001) c(2 \times 2)$ by means of combined angle-resolved photoemission and inverse photoemission spectroscopy (CARPIP) [18]. In our investigations of the clean $\mathrm{HgSe}(001) c(2 \times 2)$ surface we demonstrate that bulk valence and conduction bands are separated by a positive fundamental gap, and that band bending causes a degenerate semiconductor surface with the Fermi level being above the CBM at the surface.

All results described in this Letter were obtained on $n$-type $\left(n \approx 10^{18} \mathrm{~cm}^{-3}\right) \mathrm{HgSe}(001)$ samples prepared by cleavage in ultrahigh vacuum. Low energy electron diffraction images of the clean $\mathrm{HgSe}(001)$ surfaces always showed well-ordered $c(2 \times 2)$ reconstructions. In contrast to other II-VI and III-V compounds which cleave along (110) planes, $\mathrm{HgSe}$ produces $c(2 \times 2)$ reconstructed (001) surfaces of high quality (see also Gobrecht et al. [19]). This reconstruction is also found for $\mathrm{ZnSe}(001)$ [20] and $\mathrm{CdTe}(001)$ [21,22]. It is believed that this cleavage behavior is related to the value of the Phillips-van Vechten ionicity $f_{i}$. In $\mathrm{HgSe}$ this ionicity is near the limiting value of $f_{i}=0.78$ for a stable zinc-blende lattice [23].

Angle-resolved photoemission (ARPES) spectra were taken at room temperature with HeI radiation of a gas discharge lamp and synchrotron radiation from the DORIS III storage ring at Hamburg Synchrotron Radiation Laboratory (HASYLAB) for photon energies $10 \leq h \nu \leq$ $30 \mathrm{eV}$. The electrons were detected with an angle resolution better than $0.5^{\circ}$ by using a $180^{\circ}$ spherical analyzer mounted on a two axes goniometer. An analyzer energy resolution of 75 and $144 \mathrm{meV}$ was chosen for $\mathrm{HeI}$ and synchrotron radiation, respectively. The angleresolved inverse photoemission (ARIPES) spectra were 
taken by using a compact grating spectrometer with parallel detection of photons in the energy range of $10 \leq$ $h \nu \leq 30 \mathrm{eV}$ [18]. Electrons were focused on the sample with $\sim 1 \mathrm{~mm}^{2}$ spot size and angle divergence $<2^{\circ}$. Energy and momentum resolution are typically $400 \mathrm{meV}$ and $0.05 \AA^{-1}$. For combined angle-resolved photoemission and inverse photoemission data, a common unique energy scale is established directly by detecting the electron energy from the inverse photoemission electron gun with the photoemission electron energy analyzer. Thus, errors due to a separate determination of the Fermi level $\left(E_{\mathrm{F}}\right)$ as reference energy are avoided and band gaps can be determined without referring explicitly to $E_{\mathrm{F}}$. But, in addition, the position of the Fermi level was determined independently by photoemission from gold.

It is essential for a detailed discussion of the electronic band structure to determine the VBM. This can be done by normal emission photoemission using different photon energies. A selection of normal photoemission spectra $\left(\vartheta=0^{\circ}\right)$ taken at various photon energies corresponding to $\vec{k}_{\perp}$ wave vectors along the $\Gamma \Delta X$ direction of the bulk Brillouin zone is shown in Fig. 1. A Gaussian fit of the whole spectra in Fig. 1 around the $\Gamma$ point yields only two significant peaks for the upper valence band region (bars) clearly attributable to the two visible fundamental valence band features in the spectra. This already supports a conventional semiconductor model with degenerate valence bands at the VBM. In contrast, for a zero gap semiconductor, one would expect three nondegenerate valence bands. The VBM $\Gamma_{8}$ is characterized as the highest energy of the uppermost dispersive valence band. It is observed $0.65 \pm 0.1 \mathrm{eV}$ below $E_{\mathrm{F}}$ for $13 \mathrm{eV}$ photon energy. Assuming transitions into the same final states, emission from the CBM is also expected at $13 \mathrm{eV}$ for a zero gap semiconductor or at slightly lower photon energy for a degenerate narrow gap semiconductor. Since only very weak photoemission intensity is observed in a binding energy range of about $0.3 \mathrm{eV}$ above the $\Gamma_{8}$ maximum, an interpretation of $\mathrm{HgSe}$ as a semiconductor with nonzero gap band structure is further corroborated. Conventional semiconductors often show surface band bending. For large band bending the lowest conduction band states are possibly occupied in the surface region. In this case, emission from the CBM could be visible in the photoemission spectra. The experimental result for an energy range between 0.25 and $0.8 \mathrm{eV}$ above the VBM is enlarged in the lower part of Fig. 1. Only in the $12 \mathrm{eV}$ spectrum is an additional intensity maximum labeled $\Gamma_{6}$ at $0.42 \pm 0.05 \mathrm{eV}$ observed besides $S_{0}$, which is demonstrated by the fit results in Fig. 1. All other photoemission maxima are well reproduced by the single peak $S_{0}$ (see, e.g., the $13 \mathrm{eV}$ spectrum). Therefore the structure $\Gamma_{6}$ shows the expected behavior of the lowest conduction band of a conventional semiconductor with band bending. It should be noted here that the spectra have been carefully checked for second order-secondary tails which could not be observed. In the upper part of

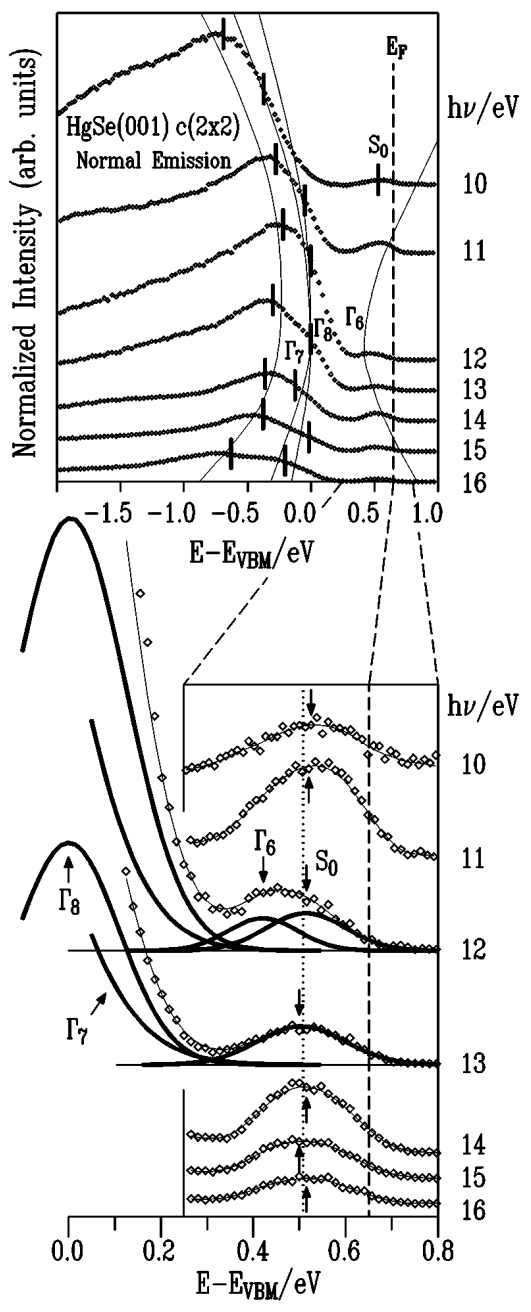

FIG. 1. Normalized photoemission spectra (raw data) of $\mathrm{HgSe}(001) c(2 \times 2)$ showing transitions near the valence band maximum $\Gamma_{8}$. Thin solid lines represent the expected dispersion for a narrow gap semiconductor. In the lower part the photoemission maximum near the Fermi energy (dashed line) with an average binding energy of $0.51 \mathrm{eV}$ (dotted line) with respect to the VBM is enlarged. Emission $\Gamma_{6}$ is attributed to the CBM. $S_{0}$ shows almost no dispersion with the Bloch wave vector $\vec{k}_{\perp}$, and is probably surface derived. Bars and arrows mark energy positions obtained by a Gaussian fit procedure. For the 12 and $13 \mathrm{eV}$ spectrum, fit results are shown in detail (solid lines).

Fig. 1 the expected dispersion of photoemission maxima near the $\Gamma$ point is shown as a guide to the eye by thin solid lines. The band dispersion guarantees the $\mathrm{CBM}$ to be visible only in the $12 \mathrm{eV}$ spectrum. Although final state effects may affect photoemission intensities at these low photon energies, the intensity enhancement at the Fermi level observed in the 11 and $14 \mathrm{eV}$ spectra may give further evidence for the crossing of the lowest conduction band. At $13 \mathrm{eV}$, emission from the conduction band may be hidden under $S_{0}$. We conclude that $\mathrm{HgSe}$ is a conventional narrow gap semiconductor with a fundamental energy gap of $0.42 \pm$ 
$0.1 \mathrm{eV}$. This is also in line with the energy position of the optical absorption at $0.4 \mathrm{eV}$ [16].

In addition, we observe a strong photoemission maximum $S_{0}$ close to the Fermi energy (dotted line in Fig. 1), showing no dispersion upon variation of the photon energy. This possibly surface-derived state is observed $0.14 \mathrm{eV}$ below $E_{\mathrm{F}}$. Figure 2 shows the dispersion of this peak along the $\bar{\Gamma} \bar{K}$ direction of the surface Brillouin zone in the occupied (upper part) and unoccupied (lower part) energy range measured by ARPES using HeI radiation and ARIPES with a constant initial state energy $E_{i}-E_{\mathrm{VBM}}=21.23 \mathrm{eV}$. In direct photoemission spectra the peak is observed only in the vicinity of the first, i.e., $k_{\|}=0 \AA^{-1}\left(\vartheta=0^{\circ}\right)$ (left side of Fig. 2) and second $\bar{\Gamma}$ point, i.e., $k_{\|}=1.46 \AA^{-1}\left(\vartheta=44^{\circ}\right)$ (right side of Fig. 2) of the surface Brillouin zone. It vanishes within
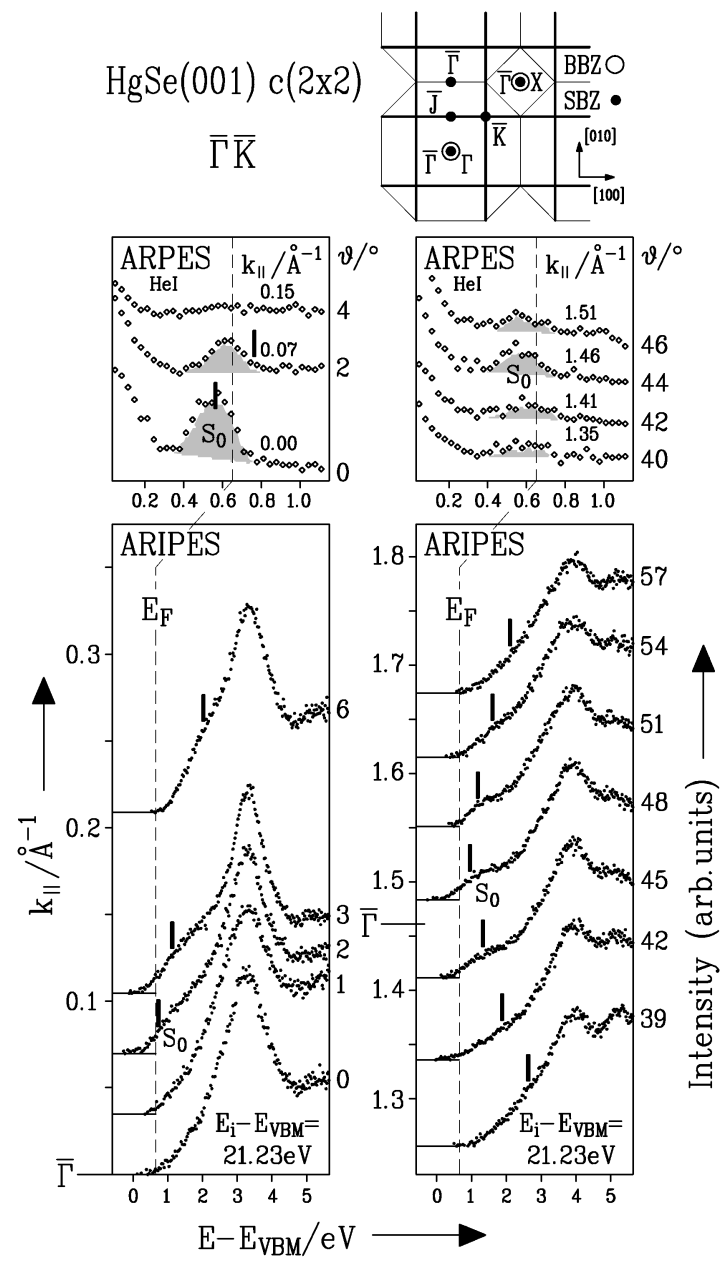

FIG. 2. Off normal direct (top) and inverse (bottom) photoemission spectra showing transitions from a surface-derived photoemission maximum $S_{0}$ in the vicinity of the first $\left(k_{\|}=\right.$ $0 \AA^{-1}$, left $)$ and second $\left(k_{\|}=1.46 \AA^{-1}\right.$, right $) \bar{\Gamma}$ point along the $\bar{\Gamma} \bar{K}$ direction of the surface Brillouin zone. Bars mark the experimental energy positions which consistently can be described by two-dimensional surface energy bands with $m^{*}=$ $0.10 m_{0}$. The inset demonstrates the periodicity of the bulk (BBZ, thin lines) and surface Brillouin zone (SBZ, thick lines). $\pm 3^{\circ}$ deviation from both of the symmetry point angles and disperses into the unoccupied part of the electronic band structure. This is proved by the appearance of a shoulder at about $k_{\|}=0.07 \AA^{-1}$ in the unoccupied energy range and the continued dispersion to higher energies (vertical bars) with increasing distance from the first $\bar{\Gamma}$ point. A similar dispersion behavior is also observed near the second $\bar{\Gamma}$ point along $\bar{\Gamma} \bar{K}$. Assuming a twodimensional parabolic and isotropic energy band structure, and taking into account the Fermi Dirac function, the dispersion of the direct and inverse photoemission peak $S_{0}$ can consistently be described in the vicinity of the $\bar{\Gamma}$ points using an effective band mass $m^{*}=0.10 m_{0}$. It should be noted here that this effective mass also describes the measured dispersion of direct and inverse photoemission maxima along the $\bar{\Gamma} \bar{J}$ direction of the surface Brillouin zone. These measurements, like other inverse photoemission experiments also showing no $\vec{k}_{\perp}$ dependence of the emission $S_{0}$, will be published elsewhere. The direct and inverse photoemission results along the $\bar{\Gamma} \bar{J}$ direction of the surface Brillouin zone exhibit no periodicity with the surface Brillouin zone, i.e., no intensity maximum at the second surface $\bar{\Gamma}$ point along $\bar{\Gamma} \bar{J}$ is observed. This clearly demonstrates that the two-dimensional photoemission structure does not correspond to the surface Brillouin zone of the reconstructed surface but to the Brillouin zone of the ideal surface (see inset in Fig. 2). Therefore, the emission $S_{0}$ cannot be attributed to a dangling bond-type surface state, and may be extended over several surface layers. Its position $0.14 \mathrm{eV}$ below $E_{\mathrm{F}}$ may explain the optical absorption edge around $0.2 \mathrm{eV} \mathrm{[16].}$

The above interpretation of the CARPIP data on $\mathrm{HgSe}$ is corroborated by experimental results of the better-known narrow gap semiconductor InAs. In Fig. 3 we compare normal emission photoemission spectra of $\operatorname{InAs}(001)(4 \times$ 2) with data obtained on $\mathrm{HgSe}$. For InAs we also observe an emission close to the Fermi level showing a very similar behavior to the structure $S_{0}$ observed on $\mathrm{HgSe}$. The energy position of the maximum near the Fermi edge does not change during the variation of the photon energy, therefore revealing no dependence on $\vec{k}_{\perp}$. Additionally, a one-to-one correspondence of the leading photoemission maxima concerning the $13 \mathrm{eV} \Gamma$ point spectrum of $\mathrm{HgSe}$ (lower part of Fig. 3) and the InAs $11 \mathrm{eV} \Gamma$ point spectrum is very obvious, also giving evidence for a typical narrow gap band structure for $\mathrm{HgSe}$. The spin orbit splitting is determined to $\Delta=0.30 \mathrm{eV}$ and $\Delta=0.34 \mathrm{eV}$ for $\mathrm{HgSe}$ and InAs, respectively. The Fermi level state in InAs also observed by Andersson et al. [24] was interpreted in the context of charge accumulation layers [25-27]. In $n$-type InAs, an accumulation of majority carriers is obtained at the surface. The resulting band bending is sufficiently high in order to shift the conduction band minimum at the surface below the Fermi level [25]. In very narrow accumulation layers, quantum size effects may appear [27], confining mobile carriers in the 


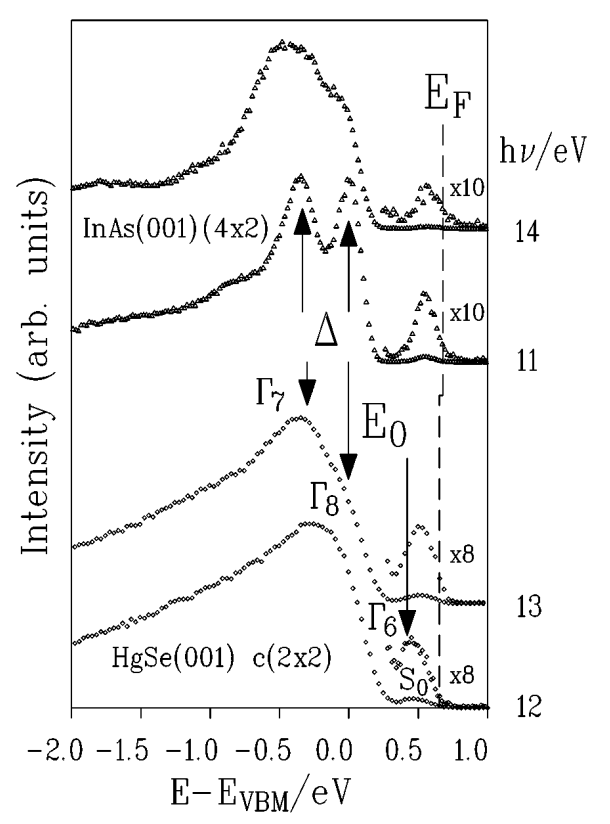

FIG. 3. Normal emission photoelectron spectra of $\mathrm{HgSe}$ in comparison with InAs displaying transitions from the top of the bulk valence band maximum. $E_{0}$ and $\Delta$ are the fundamental energy gap and spin orbit splitting having values of $E_{0}=0.42 \mathrm{eV}$ and $\Delta=0.30 \mathrm{eV}$ for $\mathrm{HgSe}$. A one-toone correspondence between the photoemission maxima gives evidence for the InAs-like narrow gap band structure for $\mathrm{HgSe}$.

space charge layer normal to the surface. Free-carrier behavior will be present parallel to the surface, resulting in two-dimensional subbands. The accumulated charge density vanishes towards the surface. This can also explain the $1 \times 1$ periodicity of photoemission structure $S_{0}$ with respect to the surface Brillouin zone of the ideal surface.

In conclusion, combined angle-resolved photoemission and inverse photoemission spectroscopy on $\mathrm{HgSe}(001)$ $c(2 \times 2)$ give evidence for a positive fundamental energy gap of $0.42 \mathrm{eV}$. In the context of the chemical trend that, with an increasing atomic number of the anion and a fixed atomic number of the cation the energy gap decreases for most of the II-V and III-V compound semiconductors, $\beta$ $\mathrm{HgS}$ is also expected to have nonzero gap band structure. Previous experiments favoring zero gap models need to be reinterpreted. Our findings call for ab initio quasiparticle calculations to describe not only the existence but also the correct magnitude of the gap. A two-dimensional surfacederived state is found close to the Fermi level above the conduction band minimum. This state may resemble a quantum size state in the accumulation space charge layer as observed on $\operatorname{InAs}(001)$ surfaces.

We would like to thank Dr. B. A. Orłowski (Polish Academy of Sciences, Warsaw) for providing the $\mathrm{HgSe}$ single crystals, and R. Schwedhelm for technical support. This research is supported by the BMBF, FR Germany (Projects No. 05622 FKB and No. 05622 KHA).
*Author to whom correspondence should be addressed.

[1] S. Groves and W. Paul, Phys. Rev. Lett. 11, 194 (1963).

[2] R. R. Galazka, W. M. Becker, and D. G. Seiler, J. Phys. Chem. Solids 32, 481 (1971).

[3] D. G. Seiler, R. R. Galazka, and W. M. Becker, Phys. Rev. B 3, 4274 (1971).

[4] A. Mycielski, J. Kossut, M. Dobrowolska, and W. Dobrowolski, J. Phys. C 15, 3293 (1982).

[5] S. H. Groves, R. N. Brown, and C. R. Pidgeon, Phys. Rev. 161, 779 (1967).

[6] R. Zallen and M. L. Slade, Solid State Commun. 8, 1291 (1970).

[7] S. Bloom and T. K. Bergstresser, Phys. Status Solidi 42, 191 (1970).

[8] H. Overhof, Phys. Status Solidi B 43, 221 (1971).

[9] S. N. Ekpenuma and C. W. Myles, J. Phys. Chem. Solids 51, 93 (1990).

[10] D. J. Chadi, J. P. Walter, M. L. Cohen, Y. Petroff, and M. Balkanski, Phys. Rev. B 5, 3058 (1972).

[11] X. Yu, L. Vanzetti, G. Haugstad, A. Raisanen, and A. Franciosi, Surf. Sci. 275, 92 (1992).

[12] M. Scrocco, J. Electron Spectrosc. Relat. Phenom. 49, 139 (1989).

[13] N. J. Shevchik, J. Tejda, M. Cardona, and D. W. Langer, Phys. Status Solidi B 59, 87 (1973).

[14] C. J. Vesely, R. L. Hengehold, and D. W. Langer, Phys. Rev. B 5, 2296 (1972).

[15] B. A. Orłowski, B. J. Kowalski, J. Bonnet, C. Hricovini, and R. Pinchaux, Vacuum 45, 199 (1994).

[16] S. Einfeldt, F. Goschenhofer, C. R. Becker, and G. Landwehr, Phys. Rev. B 51, 4915 (1995).

[17] W. Szuszkiewicz, Phys. Status Solidi B 91, 361 (1979).

[18] M. Skibowski and L. Kipp, J. Electron Spectrosc. Relat. Phenom. 68, 77 (1994).

[19] H. Gobrecht, U. Gerhardt, B. Peinemann, and A. Tausend, J. Appl. Phys. 32, 2246 (1961).

[20] W. Chen, A. Kahn, P. Soukiassian, P. S. Mangat, J. Gaines, C. Ponzoni, and D. Olego, Phys. Rev. B 49, 10790 (1994).

[21] S. Tatarenko, F. Bassani, J.C. Klein, K. Saminadayar, J. Cibert, and V.H. Etgens, J. Vac. Sci. Technol. A 12, 140 (1993).

[22] K.-U. Gawlik, J. Brügmann, S. Harm, R. Manzke, M. Skibowski, B. J. Kowalski, and B. A. Orłowski, Acta Phys. Pol. A 84, 1093 (1993).

[23] J. C. Phillips and J. A. Van Vechten, Phys. Rev. Lett. 22, 705 (1969).

[24] C. B. M. Andersson, U. O. Karlsson, L. Ilver, J. Kanski, P. O. Nilsson, L.Ö. Olsson, and M.C. Håkansson, in Proceedings of the 22nd International Conference on the Physics of Semiconductors, Vancouver, 1994, edited by D. J. Lockwood (World Scientific, Singapore, 1995), p. 489.

[25] M. Noguchi, K. Hirakawa, and T. Ikoma, Phys. Rev. Lett. 66, 2243 (1991).

[26] Y. Chen, J. C. Hermanson, and G. J. Lapeyre, Phys. Rev. B 39, 12682 (1989).

[27] T. Ando, A. B. Fowler, and F. Stern, Rev. Mod. Phys. 54, 437 (1982). 